\title{
Screening and identification of key biomarkers in bladder carcinoma: Evidence from bioinformatics analysis
}

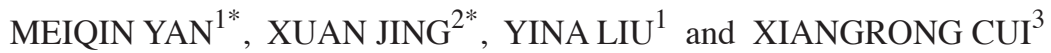 \\ ${ }^{1}$ Department of Science and Education, Children's Hospital of Shanxi and Women Health Center of Shanxi, \\ Taiyuan, Shanxi 030000; ${ }^{2}$ Clinical Laboratory, Shanxi Province People's Hospital, Taiyuan, Shanxi 030001; \\ ${ }^{3}$ Reproductive Medicine Center, Children's Hospital of Shanxi and Women Health Center of Shanxi, \\ Taiyuan, Shanxi 030000, P.R. China
}

Received October 25, 2017; Accepted May 16, 2018

DOI: $10.3892 / \mathrm{ol} .2018 .9002$

\begin{abstract}
Bladder cancer (BC) is one of the most common urogenital malignancies. However, present studies of its multiple gene interaction and cellular pathways remain unable to accurately verify the genesis and the development of BC. The aim of the present study was to investigate the genetic signatures of $\mathrm{BC}$ and identify its potential molecular mechanisms. The gene expression profiles of GSE31189 were downloaded from the Gene Expression Omnibus database. The GSE31189 dataset contained 92 samples, including 52 BC and 40 non-cancerous urothelial cells. To further examine the biological functions of the identified differentially expressed genes (DEGs), Gene Ontology (GO) and Kyoto Encyclopedia of Genes and Genomes pathway (KEGG) enrichment analyses were performed, and a protein-protein interaction (PPI) network was mapped using Cytoscape software. In total, 976 DEGs were identified in BC, including 457 upregulated genes and 519 downregulated genes. GO and KEGG pathway enrichment analyses indicated that upregulated genes were significantly enriched in the cell cycle and the negative regulation of the apoptotic process, while the downregulated genes were mainly involved in cell proliferation, cell adhesion molecules and oxidative phosphorylation pathways $(\mathrm{P}<0.05)$. From the PPI network, the 12 nodes with the highest degrees were screened as hub genes; these genes were involved in certain pathways, including the chemokine-mediated signaling
\end{abstract}

Correspondence to: Dr Xiangrong Cui, Reproductive Medicine Center, Children's Hospital of Shanxi and Women Health Center of Shanxi, 13 Xinmin Beijie, Taiyuan, Shanxi 030000, P.R. China E-mail: cuixiangrong094030@163.com

*Contributed equally

Abbreviations: DEG, differentially expressed gene; GO, gene ontology; KEGG, Kyoto Encyclopedia of Genes and Genomes; PPI, protein-protein interaction

Key words: bladder cancer, microarray analysis, differentially expressed genes, protein-protein interaction network pathway, fever generation, inflammatory response and the immune response nucleotide oligomerization domain-like receptor signaling pathway. The present study used bioinformatics analysis of gene profile datasets and identified potential therapeutic targets for BC.

\section{Introduction}

Bladder cancer (BC), the most frequently occurring urogenital malignancy of the urinary tract worldwide, results in substantial morbidity and mortality $(1,2)$. In the USA, bladder cancer is the fourth most common cancer, with 76,960 estimated new cases and 16,390 mortalities in 2016 (3). In addition to other carcinoma types, such as cervical (4), prostate (5) and ovarian cancer (6), the accumulation of inherited and somatic mutations in oncogenes and tumor suppressor genes are believed to be the reason for the occurrence, progression and metastasis of BC (7-9). Although a number of cancer-associated genes and cellular pathways have been proven to be associated with the initiation and development of BC $(10,11)$, the accuracy of early diagnosis, therapeutic and prognostic evaluation for $\mathrm{BC}$ remains low. Consequently, investigating the molecular mechanisms, including the proliferation, apoptosis and invasion of $\mathrm{BC}$ is crucial for the progress of diagnostic and treatment strategies. With the development of gene microarray technology, various advanced techniques for assessing gene expression have been widely applied in assessing tumor development and progression with lower expenses, compared with past decades $(12,13)$. In previous years, numerous gene expression profiling studies on $\mathrm{BC}$ have revealed hundreds of differentially expressed genes (DEGs) and provided substantial functional information on gene regulatory network analysis (14-16). However, comparative analysis of DEGs reported by independent research appears to rarely display substantial overlap (17), and no reliable biomarker profile discriminating cancerous from non-cancerous samples has been identified. At present, due to bioinformatics methods, the data generated by microarray technology have been analyzed in order to identify mRNA expression changes in collected urothelial cells and to examine DEGs in BC and non-cancerous urothelial cells (18). However, the interactions among DEGs, particularly pathways in the interaction network, remain to be elucidated. 
In the present study, original data (data set GSE31189) were downloaded from the Gene Expression Omnibus (GEO) (19), in order to identify DEGs between BC and non-cancerous urothelial cells. Then, functional annotation and network analyses were performed to identify DEGs. By analyzing the biological functions and networks of BC, the present study may be useful to gain a better understanding of BC development at a molecular level and explore the potential candidate biomarkers for diagnosis, prognosis and treatment.

\section{Materials and methods}

Data source. The GSE31189 gene expression profile and its corresponding platform annotation files were downloaded from the GEO database. This data set was submitted by Professor Virginia Urquidi on 3rd August 2011, last updated on 21st April 2017 and stockpiled on the GPL570 platform (HG-U133_Plus_2) Affymetrix Human Genome U133 Plus 2.0 Array (Affymetrix; Thermo Fisher Scientific, Inc., Waltham, MA, USA). This gene expression data consisted of the data of 92 samples, including $52 \mathrm{BC}$ and 40 non-cancerous urothelial cells.

Data preprocessing and screening of DEGs. The probes without annotation of the gene expression profiles were filtered using the Affy package (20), which is based on the Bioconductor principles of reproducibility, transparency and efficiency of development (21). The probe ID for each gene was then transformed into a gene symbol using the Affymetrix Human Genome U133 Plus 2.0 Array annotation data (hgu133plus2. $\mathrm{db}$ ) and Genome wide annotation for Human, version: 3.7 (org. Hs.eg.db) packages from Bioconductor (http://www. bioconductor.org/). For a gene symbol corresponding to multiple probe IDs, the average value of these probes was calculated as the representative expression level of this gene. The DEGs were screened by using the Linear Models for Microarray Analysis package in R software ( $\mathrm{R}$ x64 3.3.3) (22) with cut-off criteria of $\mathrm{P}<0.05$ and $\log _{2}$ fold-change $(\mathrm{FC}) \mid>0.5$.

Functional annotation and pathway enrichment. Gene Ontology analysis (GO) is a common useful approach for annotating genes and gene products and for identifying characteristic biological phenomena for high-throughput genome or transcriptome data $(23,24)$. To describe gene product attributes, GO provides three categories of defined terms, including biological process (BP), cellular component (CC) and molecular function (MF) categories (25). Kyoto Encyclopedia of Genes and Genomes (KEGG) is an integrated database resource for the systematic analysis of gene functions, linking genomic information with higher-order functional information (26). The two analyses were available in the Database for Annotation, Visualization and Integrated Discovery (DAVID; https://david.ncifcrf.gov/; date of access, 8/02/2018), which is a bioinformatics data resource composed of an integrated biology knowledge base and analysis tools to extract biological meanings from large quantities of genes and protein collections through a novel agglomeration algorithm (27). In the present study, GO term analysis and KEGG pathway analysis were performed using the DAVID online tool. $\mathrm{P}<0.05$ was set as the cut-off criterion.

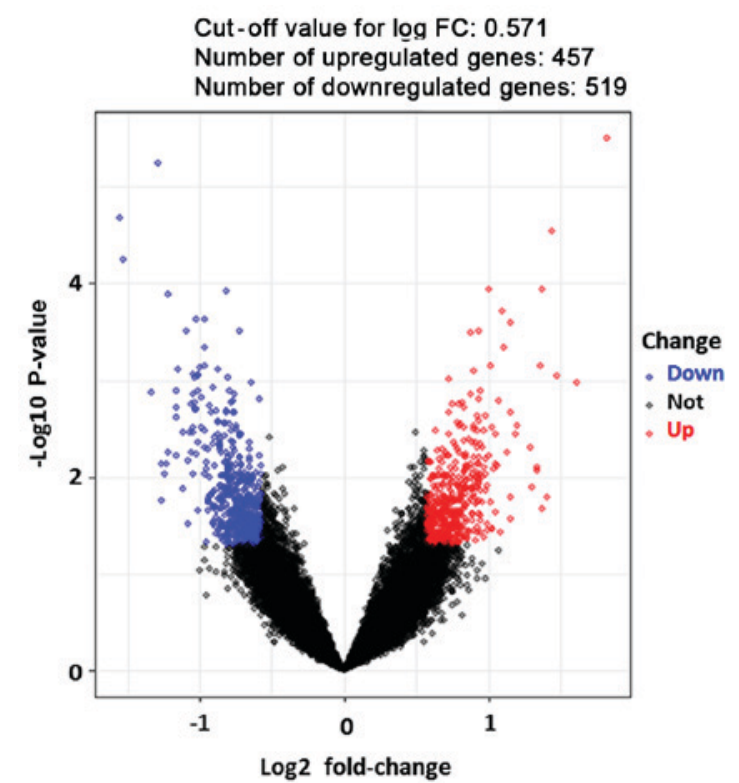

Figure 1. DEG analysis of the GSE31189 data set. DEGs were identified using the Linear Models for Microarray Analysis package. Blue indicates downregulated genes, red indicates upregulated genes and black indicates genes with unchanged expression. DEG, differentially expressed gene; FC, fold-change; down, downregulated; no, no change; up, upregulated.

PPI network construction and analysis of modules. Search Tool for the Retrieval of Interacting Genes (STRING) database (http://string-db.org/) is an online software designed to assess protein-protein interaction (PPI) information, including direct (physical) and indirect (functional) associations (28). In the present study, the DEGs were mapped using STRING to evaluate the PPI information with a combined score of $>0.4$ set as the cut-off criterion. Then, the PPI network was visualized using Cytoscape 3.5.0 (29). To screen the hub genes, a node degree of $\geq 10$ was selected as the threshold. Furthermore, the Molecular Complex Detection (MCODE) plug-in was used to screen modules of hub genes from the PPI network with degree threshold $=10$, haircut on, node score cut-off $=0.2, \mathrm{k}$-core $=2$ and maximum depth=100 (30). The genes in the significant modules were further mapped to GO terms and KEGG pathways for functional analysis.

PrognoScan database analysis. The overall survival (OS) rate of mRNA expression was assessed using an online database, PrognoScan (http://www.abren.net/PrognoScan/; keywords: NOD2, S100A9, CXCL1, CXCR2; date of access, 8/02/2018), which is a platform used for evaluating potential tumor markers and therapeutic targets. To evaluate the OS rate of patients with breast cancer, patient samples were divided into two groups by median expression [high vs. low expression; Threshold: NOD (0.87), S100A9 (0.77), CXCL1 (0.68), CXCR2 (0.55)] and analyzed using PrognoScan, with a hazard ratio with $95 \%$ confidence intervals and Cox's Proportional-Hazards Model.

\section{Results}

Data processing and DEG screening. With a criteria of the false discovery ratio $<0.05$ and $\log _{2} \mathrm{FCl} \geq 0.571$, a total of 976 DEGs (when compared with those of the noncancerous 


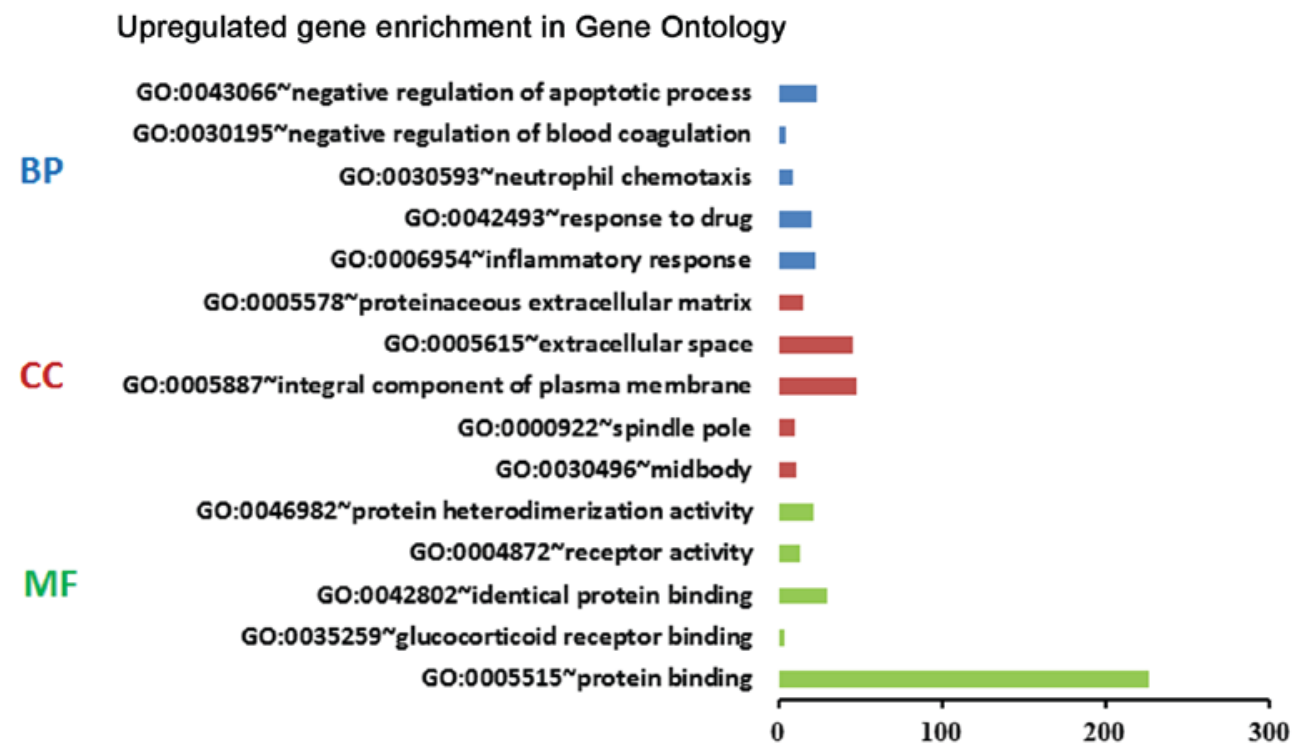

Figure 2. GO analysis of upregulated genes of the GSE31189 data set. GO, Gene Ontology; MF, molecular function; CC, cellular component; BP, biological process.

Downregulated gene enrichment in Gene Ontology

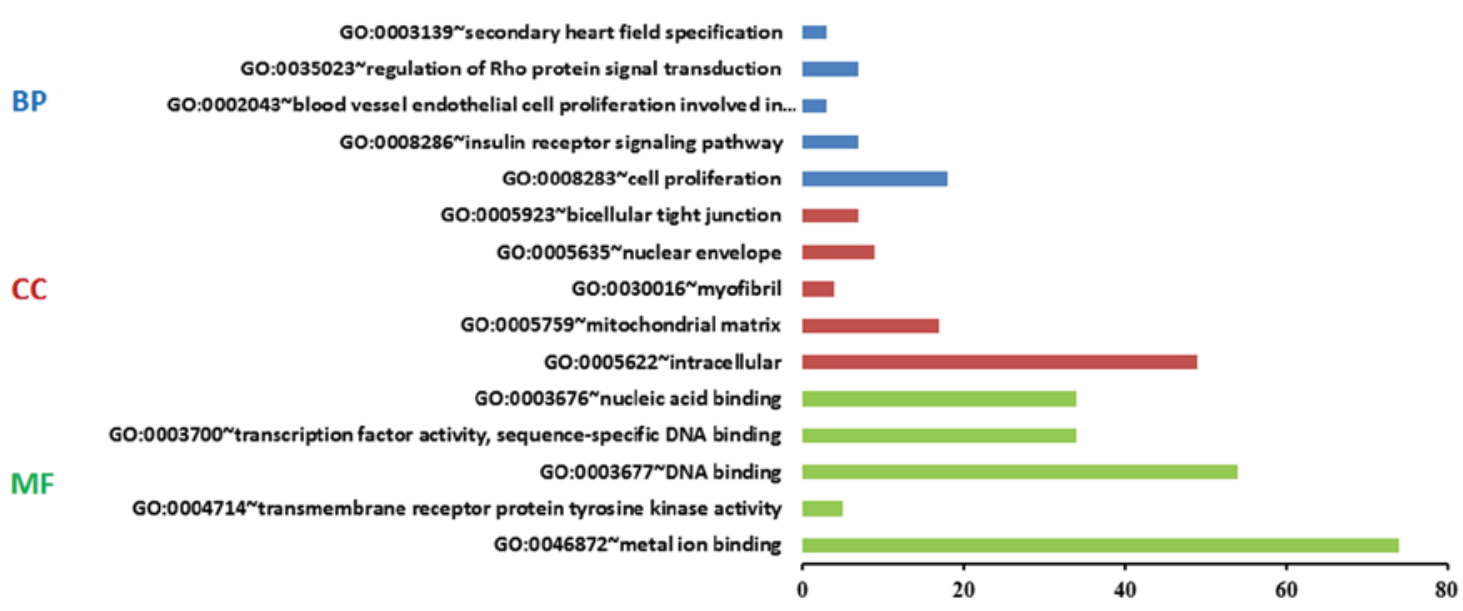

Figure 3. GO analysis of downregulated genes of the GSE31189 data set. GO, Gene Ontology; MF, molecular function; CC, cellular component; BP, biological process.

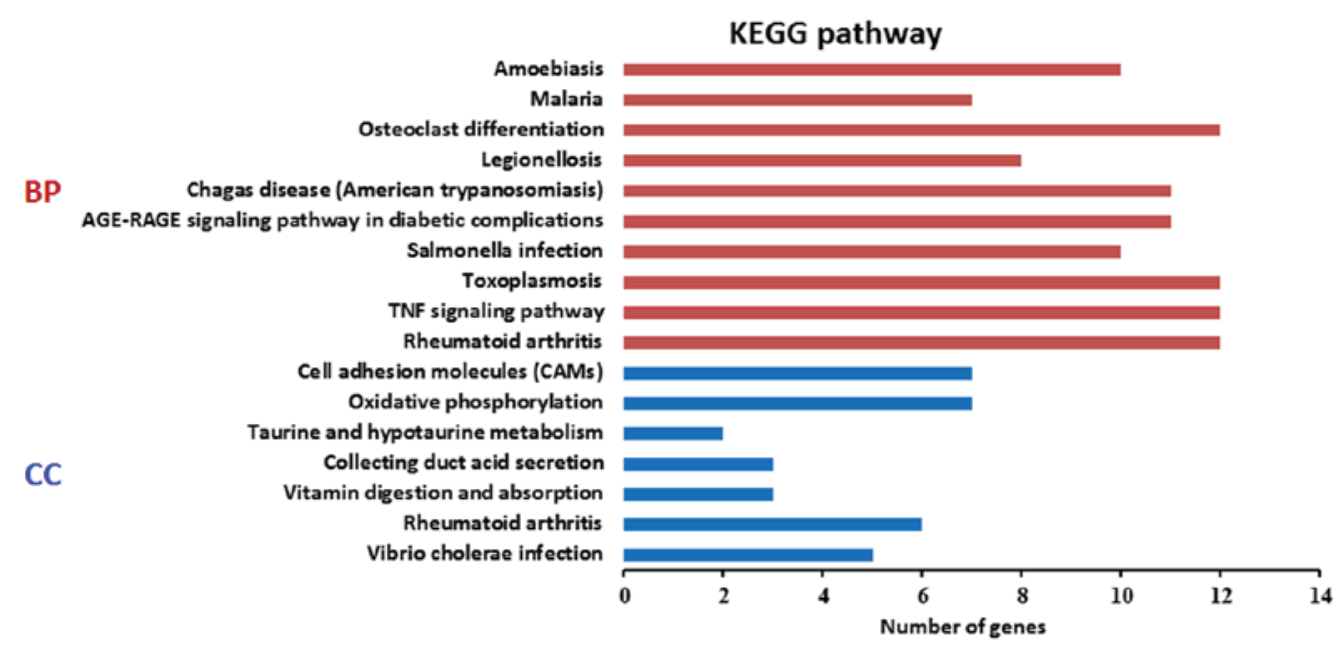

Figure 4. KEGG pathway enrichment analysis. KEGG, Kyoto Encyclopedia of Genes and Genomes; BP, biological process; CC, cellular component; TNF, tumor necrosis factor; AGE-RAGE, advanced glycation end product-receptor for AGE. 


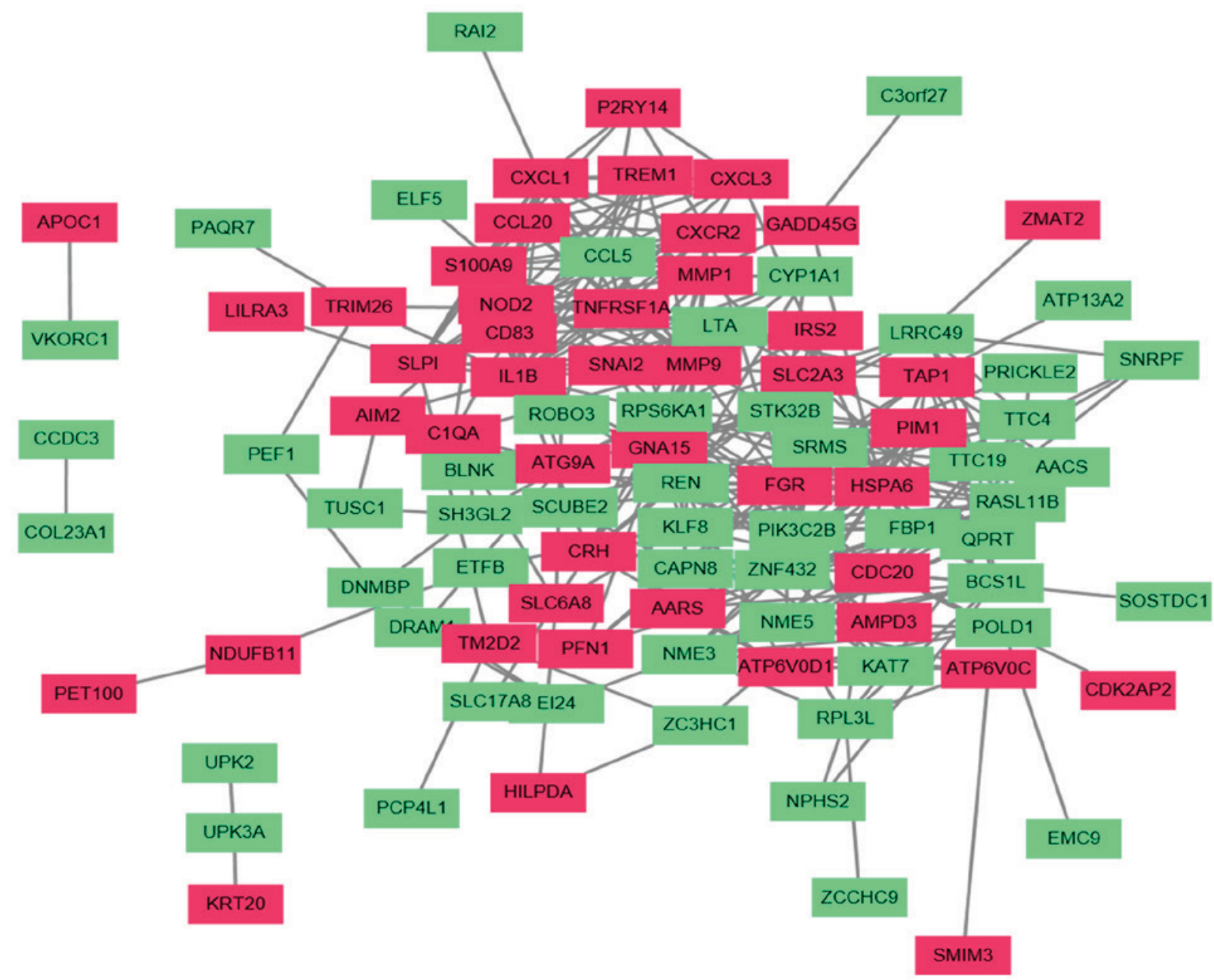

Figure 5. Protein-protein interaction network of differentially expressed genes. Red nodes denote upregulated genes, green nodes denote downregulated genes.

urothelial cells) were identified in the BC samples, including 457 upregulated genes and 519 downregulated genes (Fig. 1).

Functional enrichment of DEGs. DAVID analysis was performed to predict the potential functions and mechanisms of BC by mapping the upregulated and downregulated genes using GO terms and the KEGG pathways. The top five significant $\mathrm{GO}$ terms of the $\mathrm{BP}, \mathrm{CC}$ and $\mathrm{MF}$ categories enriched by the up- and downregulated DEGs were identified (Figs. 2 and 3). The results demonstrated that the upregulated genes were mainly involved in the negative regulation of the apoptotic process in the BP category, constituted integral components of the plasma membrane in the CC category, and were mainly involved in protein binding in the MF category (Fig. 2), whereas the downregulated genes were mainly associated with cell proliferation in the BP category, were mainly intracellular in the CC category, and were mainly involved in metal ion binding in the MF category (Fig. 3). The results for the KEGG pathway enrichment are shown in Fig. 4, which indicated that the upregulated genes were significantly enriched in amoebiasis, malaria, osteoclast differentiation, Legionellosis, Chagas disease, advanced glycation end product-receptor for AGE signaling pathway in diabetic complications, salmonella infection, toxoplasmosis, tumor necrosis factor (TNF) signaling pathway and rheumatoid arthritis pathways, while the downregulated genes were mainly enriched in cell adhesion molecules, oxidative phosphorylation, taurine and hypotaurine metabolism, collecting duct acid secretion, vitamin digestion and absorption, rheumatoid arthritis and vibrio cholerae infection pathways.

Construction of a PPI network and functional analysis for key genes. The Cytoscape tool visually constructed the PPI network with 98 nodes and 321 edges, which were predicted using STRING with a PPI score of $>0.4$ (Fig. 5). In the PPI network, 28 nodes with a degree of $\geq 10$ were regarded as key genes (Table I), including interleukin (IL)-1B, matrix metalloproteinase (MMP)9, heat shock protein family A (Hsp70) member 6, FGR proto-oncogene, Src family tyrosine kinase, ribosomal protein S6 kinase A1, TNF receptor superfamily member 1A, RAS-like family 11 member B, MMP1, chemokine ligand (CCL)5, C-X-C motif chemokine receptor 2 (CXCR2), C-X-C motif chemokine ligand 1 (CXCL1), Pim-1 proto-oncogene, serine/threonine kinase, nucleotide binding oligomerization domain containing 2 (NOD2), CCL20, src-related kinase lacking $\mathrm{C}$-terminal regulatory tyrosine and $\mathrm{N}$-terminal myristylation sites, serine threonine kinase 21B, S100 calcium binding protein A9 (S100A9), Kruppel-like factor 8, phosphatidylinositol-4-phosphate 3-kinase catalytic subunit type $2 \beta$, cluster of differentiation 83 , renin, $G$ protein 
Table I. Key nodes in the protein-protein interaction network with a degree $\geq 10$.

\begin{tabular}{lcc}
\hline Gene & Degree of change & Log $_{2}$ fold-change \\
\hline IL-1B & 23 & 1.211413 \\
MMP9 & 22 & 0.762970 \\
HSPA6 & 20 & 1.198230 \\
FGR & 18 & 0.674802 \\
RPS6KA1 & 18 & -0.692775 \\
TNFRSF1A & 17 & 0.601014 \\
RASL11B & 16 & -0.600296 \\
MMP1 & 15 & 0.743885 \\
CCL5 & 15 & -0.698948 \\
CXCR2 & 15 & 0.988178 \\
CXCL1 & 15 & 1.088843 \\
PIM1 & 14 & 0.686538 \\
NOD2 & 14 & 0.633431 \\
CCL20 & 14 & 0.677125 \\
SRMS & 13 & -0.619940 \\
STK21B & 13 & -0.598366 \\
S100A9 & 13 & 1.202516 \\
KLF8 & 13 & -0.585851 \\
PIK3c2B & 12 & -0.604130 \\
CD83 & 12 & 0.801585 \\
REN & 12 & -0.737372 \\
GNA15 & 12 & 0.647069 \\
TAP1 & 11 & 1.004478 \\
SLPI & 11 & 1.064963 \\
NME5 & 11 & -0.905805 \\
BCS1L & 10 & -0.621588 \\
CDC20 & 10 & 0.646275 \\
NME3 & 10 & -0.709424 \\
\hline
\end{tabular}

subunit $\alpha 15$, transporter 1 , ATP binding cassette subfamily B member, secretory leukocyte peptidase inhibitor, NME/NM23 family member 5, BCS homolog, ubiquinol-cytochome c reductase complex chaperone, cell division cycle 20 and NME/NM23 nucleoside diphosphate kinase 3 . One module including 12 nodes and 62 edges was obtained using MCODE (Fig. 6). The heat map indicated that the 12 genes were able to distinguish the two groups of cell samples, in that their expression was distinctly different between the two groups (Fig. 7). GO term enrichment analysis demonstrated that in the BP category, the genes in this module were significantly involved in the chemokine-mediated signaling pathway, fever generation, inflammatory responses, immune responses and the positive regulation of cell division (Table II). The genes were significantly enriched in the CC category were significantly part of the extracellular space, autophagosome, cytoplasmic vesicle, extracellular matrix and lysosome (Table II). Finally, in the MF category, analysis revealed that the genes were mainly associated with chemokine activity, IL-1 receptor binding, C-C motif receptor chemokine receptor, cytokine activity and metalloendopeptidase activity (Table II). KEGG analysis revealed that the genes were mainly enriched in the

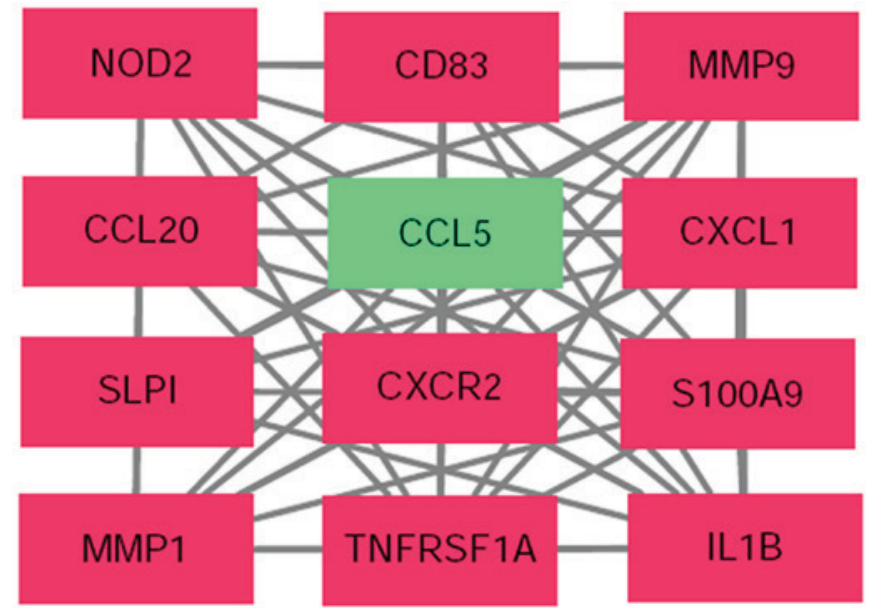

Figure 6. One significant module selected from the protein-protein interaction network. Red nodes denote upregulated genes, green nodes denote downregulated genes.

TNF signaling pathway, rheumatoid arthritis, prion diseases, cytokine-cytokine receptor interaction and NOD-like receptor signaling pathway (Table II).

Association between 12 key genes and prognosis in patients with $B C$. The PrognoScan database was used to perform the association analysis of mRNA expression and OS rate in patients with BC. As presented in Table III and Fig. 8, the high expression of NOD2 $(\mathrm{P}<0.05)$, S100A9 $(\mathrm{P}<0.001)$, CXCL1 $(\mathrm{P}<0.05)$ and $\mathrm{CXCR} 2(\mathrm{P}<0.01)$ were significantly associated with a poor prognosis in patients with $\mathrm{BC}$.

\section{Discussion}

$\mathrm{BC}$ is the ninth most common genitourinary malignancy, globally and resulted in 165,000 mortalities in 2012 (31-33). Understanding the molecular mechanism of BC is of great importance for diagnosis and treatment. Due to well-developed microarray and high-throughput sequencing technology, it is now easier to determine the general genetic alterations in the progression of diseases, and has been widely adopted to predict potential diagnosis and therapeutic targets for BC (34).

In the present study, data were extracted from the GSE31189 dataset and 457 upregulated and 519 downregulated DEGs between $\mathrm{BC}$ and normal control specimens were identified using bioinformatics analysis. The upregulated genes were enriched in the negative regulation of apoptotic processes and the positive regulation of cell division, while the downregulated genes were mainly involved in cell proliferation, bicellular tight junction, mitochondrial matrix and immune responses. Furthermore, by constructing the PPI, a number of key genes were identified that may be useful in future therapeutic studies on BC. Notably, key nodes in the PPI network and genes in the significant modules, including NOD2, S100A9, CXCL1 and $C X C R 2$, may have specific contributions to the occurrence and development of BC.

The NOD2 gene, a member of the evolutionarily conserved Nod-like receptors family, is located on chromosome 16q21 $(35,36)$. Wang et al revealed that the abnormal expression of NOD2 was highly expressed in primary liver 
Table II. Functional and pathway enrichment analysis of the genes in the module.

\begin{tabular}{|c|c|c|c|c|}
\hline Category & Term & Count & $\%$ & P-value \\
\hline GO BP & GO:0070098-chemokine-mediated signaling pathway & 3 & 40.4 & $1.09 \times 10^{-04}$ \\
\hline GO_BP & GO:0001660-fever generation & 2 & 27.0 & $2.00 \times 10^{-03}$ \\
\hline GO_BP & GO:0006954-inflammatory response & 3 & 40.4 & $3.14 \times 10^{-03}$ \\
\hline GO_BP & GO:0006955-immune response & 3 & 40.4 & $3.21 \times 10^{-03}$ \\
\hline GO_BP & GO:0051781-positive regulation of cell division & 2 & 27.0 & $5.48 \times 10^{-03}$ \\
\hline GO_CC & GO:0005615-extracellular space & 5 & 47.4 & $1.86 \times 10^{-04}$ \\
\hline GO_CC & GO:0005776-autophagosome & 2 & 27.0 & $1.67 \times 10^{-02}$ \\
\hline GO_CC & GO:0031410-cytoplasmic vesicle & 2 & 27.0 & $3.19 \times 10^{-02}$ \\
\hline GO_CC & GO:0031012-extracellular matrix & 2 & 27.0 & $3.66 \times 10^{-02}$ \\
\hline GO_CC & GO:0005764-lysosome & 2 & 27.0 & $4.73 \times 10^{-02}$ \\
\hline GO_MF & GO:0008009-chemokine activity & 3 & 40.4 & $1.16 \times 10^{-04}$ \\
\hline GO_MF & GO:0005149-interleukin-1 receptor binding & 2 & 27.0 & $4.78 \times 10^{-03}$ \\
\hline GO_MF & GO:0048020-C-C motif receptor chemokine receptor binding & 2 & 27.0 & $1.01 \times 10^{-02}$ \\
\hline GO_MF & GO:0005125-cytokine activity & 2 & 27.0 & $4.28 \times 10^{-02}$ \\
\hline GO_MF & GO:0004222-metalloendopeptidase activity & 2 & 27.0 & $4.95 \times 10^{-02}$ \\
\hline KEGG_PATHWAY & ecb04668: Tumor necrosis factor signaling pathway & 6 & 80.9 & $4.39 \times 10^{-09}$ \\
\hline KEGG_PATHWAY & ecb05323: Rheumatoid arthritis & 5 & 67.4 & $3.04 \times 10^{-07}$ \\
\hline KEGG_PATHWAY & ecb05020: Prion diseases & 3 & 40.4 & $3.42 \times 10^{-04}$ \\
\hline KEGG_PATHWAY & ecb04060: Cytokine-cytokine receptor interaction & 4 & 53.9 & $5.48 \times 10^{-04}$ \\
\hline KEGG_PATHWAY & $\begin{array}{l}\text { ecb04621: Nucleotide oligomerization domain-like receptor } \\
\text { signaling pathway }\end{array}$ & 3 & 40.4 & $8.04 \times 10^{-04}$ \\
\hline
\end{tabular}

GO, Gene Ontology; BP, biological process; CC, cellular component; MF, molecular function; KEGG, Kyoto Encyclopedia of Genes and Genomes. The count was the number of enriched genes in each term. If there were $>5$ terms enriched in this category, the top five terms were selected according to their P-value.

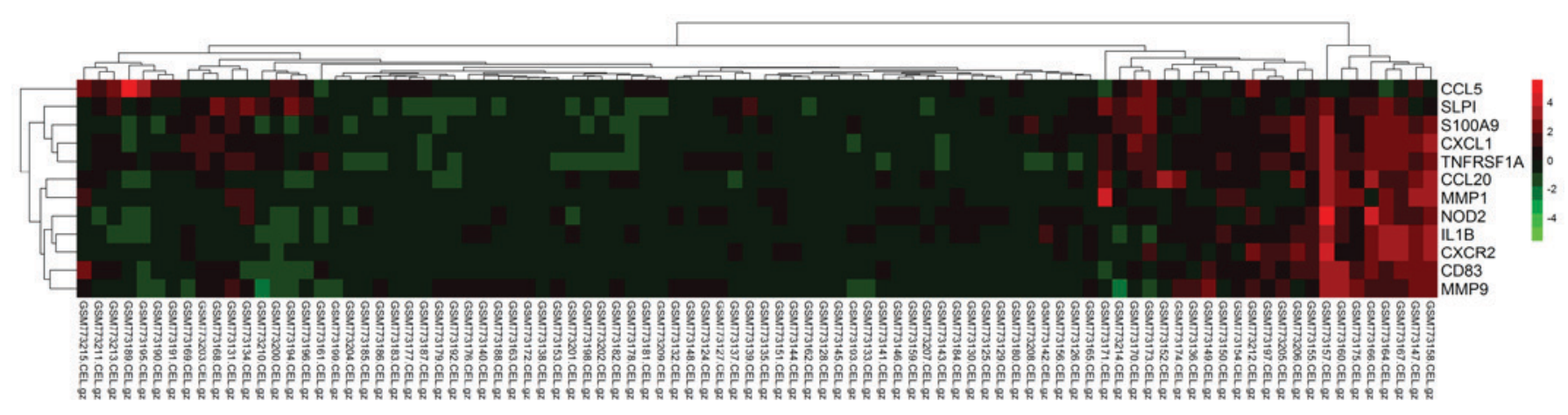

Figure 7. Hub genes expression heat map in the GSE31189 data set. Red indicates upregulation and green indicates downregulation.

tumor types, which was associated with a shorter median survival time (36). In addition, in the present study, a higher NOD2 mRNA expression was identified in BC, which was associated with a shorter OS rate.

S100A9, a member of the S100 family of calcium-binding proteins, is primarily detected in neutrophil granulocytes and known to serve a function in the innate immune system $(37,38)$. Previous studies have demonstrated that the abnormal overexpression of S100A9 is an unfavorable prognostic factor for carcinogenesis and prognosis in various neoplasms, such as hypopharyngeal and bladder cancer (39-43). Additionally, S100A9 is associated with colorectal carcinoma progression and contributes to colorectal carcinoma cell survival and migration via the $\mathrm{Wnt} / \beta$-catenin pathway (37). In the present study, S100A9 was revealed to be differentially expressed in $\mathrm{BC}$ and non-cancerous urothelial cells and was identified as a key node in the PPI network constructed using DEGs between these two groups. The results additionally demonstrated that a high S100A9 expression was associated with a shorter OS rate. Therefore, this gene may be an essential marker for the diagnosis and prognosis of $\mathrm{BC}$.

CXCL1, a member of the CXC chemokine family, was originally characterized by Wang et al (44) and is known to promote the proliferation of melanoma cells. Previous studies have demonstrated that CXCL1 may be associated with tumor epithelial-stromal interactions that facilitate tumor 
Table III. Association between mRNA expression of enriched genes and overall survival in patients with bladder cancer.

\begin{tabular}{lllcc}
\hline Gene name & \multicolumn{1}{c}{ End point } & PROBE ID & n & Cox P-value \\
\hline CCL5 & Overall survival rate & ILMN_1773352 & 165 & 0.112459 \\
SLPI & Overall survival rate & ILMN_1669650 & 165 & 0.225693 \\
S100A9 & Overall survival rate & ILMN_1714991 & 165 & 0.000119 \\
CXCL1 & Overall survival rate & ILMN_1787897 & 165 & 0.013803 \\
TNFRSF1A & Overall survival rate & ILMN_1685005 & 165 & 0.732444 \\
CCL20 & Overall survival rate & ILMN_1657234 & 165 & 0.636372 \\
MMP1 & Overall survival rate & ILMN_1726448 & 165 & 0.279756 \\
NOD2 & Overall survival rate & ILMN_1762594 & 165 & 0.025039 \\
IL1B & Overall survival rate & ILMN_1775501 & 165 & 0.374688 \\
CXCR2 & Overall survival rate & ILMN_1783085 & 165 & 0.006080 \\
CD83 & Overall survival rate & ILMN_1780582 & 165 & 0.169956 \\
MMP1 & Overall survival rate & ILMN_1796316 & 165 & 0.114052 \\
\hline
\end{tabular}
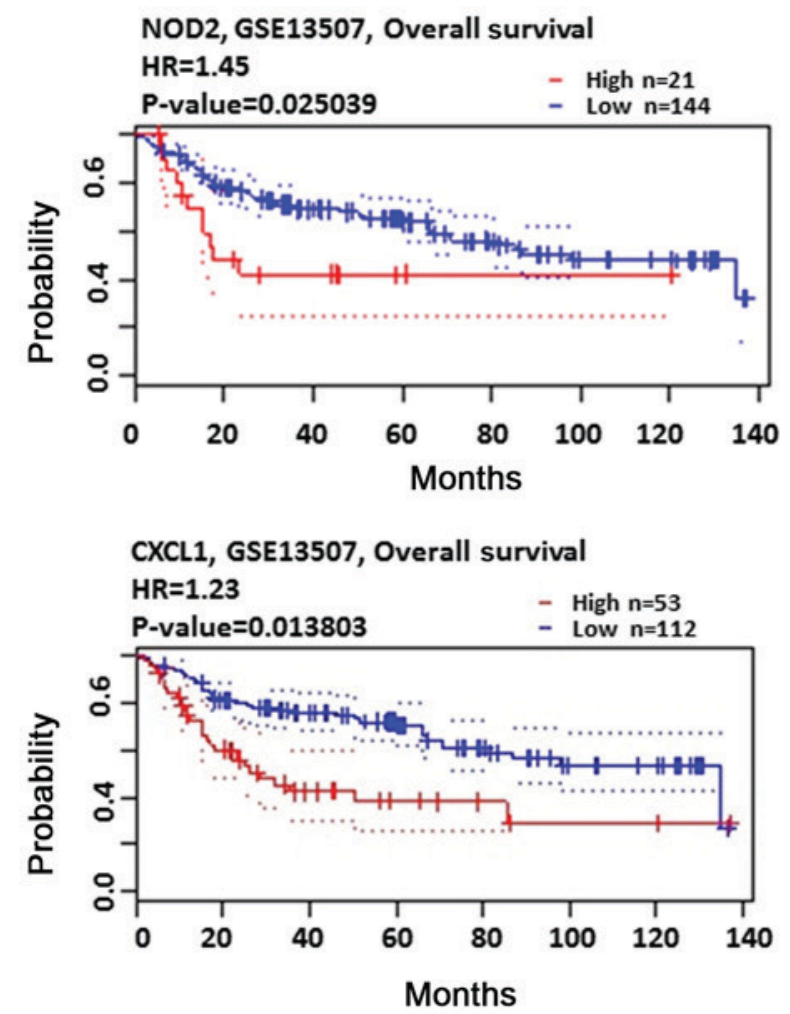
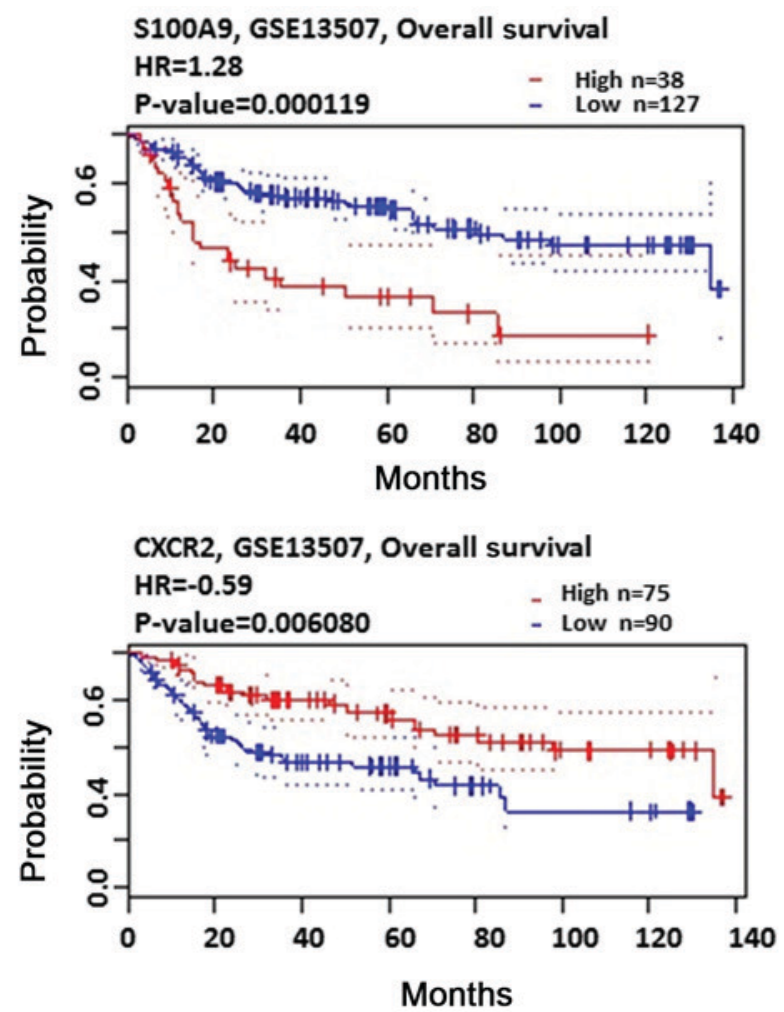

Figure 8. Association between mRNA expression and overall survival rate in patients with bladder cancer (using the PrognoScan database). $\mathrm{P}<0.05$ was used as the threshold. NOD2, nucleotide binding oligomerization domain containing 2; S100A9, S100 calcium binding protein A9; CXCL1, C-X-C motif chemokine ligand 1; CXCR2, C-X-C motif chemokine receptor 2; HR, hazard ratio.

growth and invasion (45-47). In addition, Wang et al (48) revealed that $\mathrm{CXCL1}$ derived from tumor-associated lymphatic endothelial cells drives gastric cancer cells into the lymphatic system by activating integrin $\beta 1 /$ focal adhesion kinase/protein kinase B (Akt) signaling. Furthermore, Kawanishi et al (49) revealed that CXCL1 may modulate the invasive abilities of $\mathrm{BC}$ cells, and therefore is a potential candidate biomarker and therapeutic target for invasive $\mathrm{BC}$. In the present study, a higher CXCL1 mRNA expression was identified in patients with BC in the GSE31189 gene expression profile. Furthermore, PrognoScan analysis results revealed that high CXCL1 expression was significantly associated with a shorter OS rate. Due to these findings, the expression level of CXCL1 may be a useful prognostic marker of $\mathrm{BC}$.

CXCR2, the co-receptor of IL-8 and CXCL1, is an important therapeutic target in a number of solid tumor types, including lung, breast, prostate, ovarian, colorectal and liver cancer (50-55). Xu et al (56) revealed that CXCR2 may promote breast cancer metastasis and chemoresistance 
via the suppression of AKT serine/threonine kinase 1 and activation of cyclooxygenase 2. Inhibition of CXCR2 may reduce the activity of breast cancer stem cells and improve the survival of human epidermal growth factor receptor 2 (HER2)-positive patients in combination with HER2-target chemotherapies (57). Furthermore, Gao et al (58) demonstrated that the CXCL5/CXCR2 axis may promote BC cell migration and invasion by activating the phosphoinositide 3-kinase/Akt-induced upregulation of MMP2/MMP9. In the present study, CXCR2 was increased in BC compared with normal specimens, similar with the previous studies. However, the BC patients with higher mRNA levels of CXCR2 were predicted to have a better OS rate. As few studies have focused on CXCR2, the underlying function of $\mathrm{CXCR} 2$ requires further research.

Altogether, the DEGs identified in the BC urothelial cells when compared with the normal controls may be involved in tumorigenesis. The key nodes identified in the PPI network constructed with these DEGs and genes involved in the significant module, including NOD2, S100A9 and CXCL1, may be important in the development of $\mathrm{BC}$, and may provide valuable clues in order to investigate the pathogenesis of $\mathrm{BC}$. However, further biological experimental evidence is required in order to confirm the function of the identified gene in BC.

\section{Acknowledgements}

Not applicable.

\section{Funding}

The present study was supported by the Scientific Research Project of Shanxi Provincial Department of Health (grant no. 201601070).

\section{Availability of data and materials}

All data generated or analyzed during this study are included in this published article.

\section{Authors' contributions}

$\mathrm{XJ}$ and $\mathrm{XC}$ participated in the design of the present study, performed the statistical analysis and drafted the manuscript. MY and YL performed the study and collected background information and data. All authors read and approved the final manuscript.

\section{Ethics approval and consent to participate}

Not applicable.

\section{Patient consent for publication}

Not applicable.

\section{Competing interests}

The authors declare that there are no competing interests.

\section{References}

1. Hao L, Zhao Y, Li ZG, He HG, Liang Q, Zhang ZG, Shi ZD, Zhang PY and Han CH: Tumor necrosis factor-related apoptosis-inducing ligand inhibits proliferation and induces apoptosis of prostate and bladder cancer cells. Oncol Lett 13: 3638-3640, 2017.

2. Li X, Ma X, Tang L, Wang B, Chen L, Zhang F and Zhang X: Prognostic value of neutrophil-to-lymphocyte ratio in urothelial carcinoma of the upper urinary tract and bladder: A systematic review and meta-analys. Oncotarget 8: 62681-62692, 2016.

3. Peng M, Huang Y, Tao T, Peng CY, Su Q, Xu W, Darko KO, Tao X and Yang X: Metformin and gefitinib cooperate to inhibit bladder cancer growth via both AMPK and EGFR pathways joining at Akt and Erk. Sci Rep 6: 28611, 2016.

4. Kim HJ, Eoh KJ, Kim LK, Nam EJ, Yoon SO, Kim KH, Lee JK, Kim SW and Kim YT: The long noncoding RNA HOXA11 antisense induces tumor progression and stemness maintenance in cervical cancer. Oncotarget 7: 83001-83016, 2016.

5. Luo G, Wang M, Wu X, Tao D, Xiao X, Wang L, Min F, Zeng F and Jiang G: Long non-coding RNA meg3 inhibits cell proliferation and induces apoptosis in prostate cancer. Cell Physiol Biochem 37: 2209-2220, 2015.

6. Huang S, Qing C, Huang Z and Zhu Y: The long non-coding RNA CCAT2 is up-regulated in ovarian cancer and associated with poor prognosis. Diagn Pathol 11: 49, 2016.

7. Cui X, Jing X, Long C, Yi Q, Tian J and Zhu J: Accuracy of the urine UCA1 for diagnosis of bladder cancer: A meta-analysis. Oncotarget 8: 35222-35233, 2017.

8. Cui $\mathrm{X}$, Jing $\mathrm{X}$ and $\mathrm{Wu} \mathrm{X}$ : The prognostic value of long non coding RNAs in cervical cancer: A meta-analysis. Oncotarget 8: 62470-62477, 2017.

9. Jing X, Liang H, Cui X, Han C, Hao C and Huo K: Long noncoding RNA CCAT2 can predict metastasis and a poor prognosis: A meta-analysis. Clin Chim Acta 468: 159-165, 2017.

10. Cui X, Jing X, Yi Q, Long C, Tan B, Li X, Chen X, Huang Y, Xiang Z, Tian J and Zhu J: Systematic analysis of gene expression alterations and clinical outcomes of STAT3 in cancer. Oncotarget 9: 3198-3213, 2018.

11. Cui X, Jing X, Yi Q, Long C, Tian J and Zhu J: Clinicopathological and prognostic significance of SDC1 overexpression in breast cancer. Oncotarget 8: 111444-111455, 2017.

12. Guo Y, Sheng Q, Li J, Ye F, Samuels DC and Shyr Y: Large scale comparison of gene expression levels by microarrays and RNAseq using TCGA data. PLoS One 8: e71462, 2013.

13. Senchenko VN, Kisseljova NP, Ivanova TA, Dmitriev AA, Krasnov GS, Kudryavtseva AV, Panasenko GV, Tsitrin EB, Lerman MI, Kisseljov FL, et al: Novel tumor suppressor candidates on chromosome 3 revealed by NotI-microarrays in cervical cancer. Epigenetics 8: 409-420, 2013.

14. Chen G, Li Y, Su Y, Zhou L, Zhang H, Shen Q, Du C, Li H, Wen Z, Xia Y and Tang W: Identification of candidate genes for necrotizing enterocolitis based on microarray data. Gene 661: 152-159, 2018.

15. Shu B, Fang Y, He W, Yang J and Dai C: Identification of macrophage-related candidate genes in lupus nephritis using bioinformatics analysis. Cell Signal 46: 43-51, 2018.

16. Shao K, Shen LS, Li HH, Huang $S$ and Zhang Y: Systematic-analysis of mRNA expression profiles in skeletal muscle of patients with type II diabetes: The glucocorticoid was central in pathogenesis. J Cell Physiol 233: 4068-4076, 2018.

17. Chen J, Ding J, Wang Z, Zhu J, Wang X and Du J: Identification of downstream metastasis-associated target genes regulated by LSD1 in colon cancer cells. Oncotarget 8: 19609-19630, 2017.

18. Urquidi V, Goodison S, Cai Y, Sun Y and Rosser CJ: A candidate molecular biomarker panel for the detection of bladder cancer. Cancer Epidemiol Biomarkers Prev 21: 2149-2158, 2012.

19. Edgar R, Domrachev M and Lash AE: Gene expression omnibus: NCBI gene expression and hybridization array data repository. Nucleic Acids Res 30: 207-210, 2002.

20. Gautier L, Cope L, Bolstad BM and Irizarry RA: Affy-analysis of Affymetrix GeneChip data at the probe level. Bioinformatics 20: 307-315, 2004

21. Carvalho BS and Irizarry RA: A framework for oligonucleotide microarray preprocessing. Bioinformatics 26 : 2363-2367, 2010.

22. Gentleman RC, Carey VJ, Bates DM, Bolstad B, Dettling M, Dudoit S, Ellis B, Gautier L, Ge Y, Gentry J, et al: Bioconductor: Open software development for computational biology and bioinformatics. Genome Biol 5: R80, 2004. 
23. Gene Ontology Consortium: The Gene Ontology (GO) project in 2006. Nucleic Acids Res 34 (Database Issue): D322-D326, 2006.

24. Ashburner M, Ball CA, Blake JA, Botstein D, Butler H, Cherry JM, Davis AP, Dolinski K, Dwight SS, Eppig JT, et al: Gene ontology: Tool for the unification of biology. The Gene Ontology Consortium. Nat Genet 25: 25-29, 2000.

25. Harris MA, Clark J, Ireland A, Lomax J, Ashburner M, Foulger R, Eilbeck K, Lewis S, Marshall B, Mungall C, et al: The Gene Ontology (GO) database and informatics resource. Nucleic Acids Res 32 (Database Issue): D258-D261, 2004.

26. Kanehisa M, Araki M, Goto S, Hattori M, Hirakawa M, Itoh M, Katayama T, Kawashima S, Okuda S, Tokimatsu T and Yamanishi Y: KEGG for linking genomes to life and the environment. Nucleic Acids Res 36 (Database Issue): D480-D484, 2008

27. Huang da W, Sherman BT and Lempicki RA: Systematic and integrative analysis of large gene lists using DAVID bioinformatics resources. Nat Protoc 4: 44-57, 2009.

28. Szklarczyk D, Franceschini A, Wyder S, Forslund K, Heller D, Huerta-Cepas J, Simonovic M, Roth A, Santos A, Tsafou KP, et al: STRING v10: Protein-protein interaction networks, integrated over the tree of life. Nucleic Acids Res 43 (Database Issue): D447-D452, 2015.

29. Smoot ME, Ono K, Ruscheinski J, Wang PL and Ideker T: Cytoscape 2.8: New features for data integration and network visualization. Bioinformatics 27: 431-432, 2011.

30. Wishart DS, Knox C, Guo AC, Shrivastava S, Hassanali M, Stothard P, Chang Z and Woolsey J: DrugBank: A comprehensive resource for in silico drug discovery and exploration. Nucleic Acids Res 34 (Database Issue): D668-D672, 2006.

31. Cui X, Jing X, Long C, Tian J and Zhu J: Long noncoding RNA MEG3, a potential novel biomarker to predict the clinical outcome of cancer patients: A meta-analysis. Oncotarget 8 : 19049-19056, 2017.

32. Gnesin S, Mitsakis P, Cicone F, Deshayes E, Dunet V, Gallino AF, Kosinski M, Baechler S, Buchegger F, Viertl D and Prior JO First in-human radiation dosimetry of ${ }^{68} \mathrm{Ga}$-NODAGA-RGDyK. EJNMMI Res 7: 43, 2017.

33. Cohen T, Ricchiuti D and Memo M: Bladder cancer that metastasized to the skin: A unique presentation that signifies poor prognosis. Rev Urol 19: 67-71, 2017.

34. Zhang Y, Fang L, Zang Y and Xu Z: Identification of core genes and key pathways via integrated analysis of gene expression and DNA methylation profiles in bladder cancer. Med Sci Monit 24: 3024-3033, 2018.

35. Liu J, He C, Xu Q, Xing C and Yuan Y: NOD2 polymorphisms associated with cancer risk: A meta-analysis. PLoS One 9: e89340, 2014.

36. Wang X, Yang C, Liao X, Han C, Yu T, Huang K, Yu L, Qin W, Zhu G, Su H, et al: NLRC and NLRX gene family mRNA expression and prognostic value in hepatocellular carcinoma. Cancer Med 6: 2660-2672, 2017.

37. Duan L, Wu R, Ye L, Wang H, Yang X, Zhang Y, Chen X, Zuo G, Zhang Y, Weng Y, et al: S100A8 and S100A9 are associated with colorectal carcinoma progression and contribute to colorectal carcinoma cell survival and migration via $\mathrm{Wnt} / \beta$-catenin pathway. PLoS One 8: e62092, 2013.

38. Källberg E, Vogl T, Liberg D, Olsson A, Björk P, Wikström P, Bergh A, Roth J, Ivars F and Leanderson T: S100A9 interaction with TLR4 promotes tumor growth. PLoS One 7: e34207, 2012.

39. De Veirman K, De Beule N, Maes K, Menu E, De Bruyne E, De Raeve H, Fostier K, Moreaux J, Kassambara A, Hose D, et al: Extracellular S100A9 protein in bone marrow supports multiple myeloma survival by stimulating angiogenesis and cytokine secretion. Cancer Immunol Res 5: 839-846, 2017.

40. Yasar O, Akcay T, Obek C and Turegun FA: Significance of S100A8, S100A9 and calprotectin levels in bladder cancer. Scand J Clin Lab Invest 77: 437-441, 2017.

41. Wu P, Quan H, Kang J, He J, Luo S, Xie C, Xu J, Tang Y and Zhao S: Downregulation of calcium-binding protein S100A9 inhibits hypopharyngeal cancer cell proliferation and invasion ability through inactivation of NF-кB signaling. Oncol Res 25: 1479-1488, 2017.

42. Yang Q, Li X, Chen H, Cao Y, Xiao Q, He Y, Wei J and Zhou J: IRF7 regulates the development of granulocytic myeloid-derived suppressor cells through S100A9 transrepression in cancer. Oncogene 36: 2969-2980, 2017.
43. Lim SY, Yuzhalin AE, Gordon-Weeks AN and Muschel RJ: Tumor-infiltrating monocytes/macrophages promote tumor invasion and migration by upregulating S100A8 and S100A9 expression in cancer cells. Oncogene 35: 5735-5745, 2016.

44. Wang D, Yang W, Du J, Devalaraja MN, Liang P, Matsumoto K, Tsubakimoto K,Endo T and Richmond A: MGSA/GRO-mediated melanocyte transformation involves induction of Ras expression. Oncogene 19: 4647-4659, 2000.

45. Verbeke H, Struyf S, Laureys G and Van Damme J: The expression and role of CXC chemokines in colorectal cancer. Cytokine Growth Factor Rev 22: 345-358, 2011.

46. Miyake M, Lawton A, Goodison S, Urquidi V, Gomes-Giacoia E, Zhang G, Ross S, Kim J and Rosser CJ: Chemokine (C-X-C) ligand 1 (CXCL1) protein expression is increased in aggressive bladder cancers. BMC Cancer 13: 322, 2013.

47. Han KQ, Han H, He XQ, Wang L, Guo XD, Zhang XM, Chen J, Zhu QG, Nian H, Zhai XF and Jiang MW: Chemokine CXCL1 may serve as a potential molecular target for hepatocellular carcinoma. Cancer Med 5: 2861-2871, 2016.

48. Wang Z, Wang Z, Li G, Wu H, Sun K, Chen J, Feng Y, Chen C, Cai S, Xu J and He Y: CXCL1 from tumor-associated lymphatic endothelial cells drives gastric cancer cell into lymphatic system via activating integrin $\beta 1 / F A K / A K T$ signaling. Cancer Lett 385 : 28-38, 2017

49. Kawanishi H, Matsui $\mathrm{Y}$, Ito $\mathrm{M}$, Watanabe J, Takahashi T, Nishizawa K, Nishiyama H, Kamoto T, Mikami Y, Tanaka Y, et al: Secreted CXCL1 is a potential mediator and marker of the tumor invasion of bladder cancer. Clin Cancer Res 14: 2579-2587, 2008

50. Cong L, Qiu ZY, Zhao Y, Wang WB, Wang CX, Shen HC and Han JQ: Loss of $\beta$-arrestin-2 and activation of CXCR2 correlate with lymph node metastasis in non-small cell lung cancer. J Cancer 8: 2785-2792, 2017.

51. Devapatla B, Sharma A and Woo S: CXCR2 inhibition combined with sorafenib improved antitumor and antiangiogenic response in preclinical models of ovarian cancer. PLoS One 10: e0139237, 2015.

52. Zhang T, Tseng C, Zhang Y, Sirin O, Corn PG, Li-Ning-Tapia EM, Troncoso P, Davis J, Pettaway C, Ward J, et al: CXCL1 mediates obesity-associated adipose stromal cell trafficking and function in the tumour microenvironment. Nat Commun 7: 11674, 2016.

53. Ignacio RM, Kabir SM, Lee ES, Adunyah SE and Son DS: NF- $\kappa$ B-mediated CCL20 reigns dominantly in CXCR2-driven ovarian cancer progression. PLoS One 11: e0164189, 2016.

54. Zhao J, Ou B, Feng H, Wang P, Yin S, Zhu C, Wang S, Chen C, Zheng M, Zong Y, et al: Overexpression of CXCR2 predicts poor prognosis in patients with colorectal cancer. Oncotarget 8: 28442-28454, 2017.

55. Ding D, Zhang Y, Yang R, Wang X, Ji G, Huo L, Shao Z and Li X: miR-940 suppresses tumor cell invasion and migration via regulation of CXCR2 in hepatocellular carcinoma. Biomed Res Int 2016: 7618342, 2016

56. Xu H, Lin F, Wang Z, Yang L, Meng J, Ou Z, Shao Z, Di G and Yang G: CXCR 2 promotes breast cancer metastasis and chemoresistance via suppression of AKT1 and activation of COX2. Cancer Lett 412: 69-80, 2018

57. Singh JK, Farnie G, Bundred NJ, Simões BM, Shergill A, Landberg G, Howell SJ and Clarke RB: Targeting CXCR1/2 significantly reduces breast cancer stem cell activity and increases the efficacy of inhibiting HER2 via HER2-dependent and -independent mechanisms. Clin Cancer Res 19: 643-656, 2013.

58. Gao Y, Guan Z, Chen J, Xie H, Yang Z, Fan J, Wang X and Li L: CXCL5/CXCR2 axis promotes bladder cancer cell migration and invasion by activating PI3K/AKT-induced upregulation of MMP2/MMP9. Int J Oncol 47: 690-700, 2015.

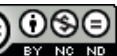

This work is licensed under a Creative Commons Attribution-NonCommercial-NoDerivatives 4.0 International (CC BY-NC-ND 4.0) License. 